\title{
Neuropsychic Disorders Unmasking the Coexistence of Fahr Syndrome and Neuromeningeal Cryptococcosis
}

\author{
Mikaila Kaboré1 ${ }^{*}$, Issa Konaté1,2, Yacouba Cissoko1,2, Souleymane P. Coulibaly ${ }^{2,3}$, \\ Jean-Paul Dembélé1,2, Assetou Fofana1, Mariam Soumaré1, Hermine Méli1, Dramane Sogoba1, \\ Oumar Magassouba1, Adama Sissoko²,4, Mohamed Aly Cissé1, Abdoulaye Zaré1, \\ Bintou Coulibaly'1, Fodé Kouyaté1, Moïse Nguemeni' ${ }^{1}$, Sounkalo Dao ${ }^{1,2,5}$ \\ ${ }^{1}$ Infectious Diseases Department, Point “G” University Teaching Hospital, Bamako, Mali \\ ${ }^{2}$ Faculty of Medicine and Odontostomatology, University of Sciences, Techniques and Technologies of Bamako (USTTB), \\ Bamako, Mali \\ ${ }^{3}$ Psychiatry Department, Point “G” University Teaching Hospital, Bamako, Mali \\ ${ }^{4}$ Neurology Department, Point “G” University Teaching Hospital, Bamako, Mali \\ ${ }^{5}$ Serefo Program, University of Sciences, Techniques and Technologies of Bamako (USTTB), Bamako, Mali \\ Email: *mikailakab@gmail.com
}

How to cite this paper: Kaboré, M., Konaté, I., Cissoko, Y., Coulibaly, S.P., Dembélé, J.-P., Fofana, A., Soumaré, M., Méli, H., Sogoba, D., Magassouba, O., Sissoko, A., Cissé, M.A., Zaré, A., Coulibaly, B., Kouyaté, F., Nguemeni, M. and Dao, S. (2020) Neuropsychic Disorders Unmasking the Coexistence of Fahr Syndrome and Neuromeningeal Cryptococcosis. Case Reports in Clinical Medicine, 9, 329-334.

https://doi.org/10.4236/crcm.2020.911046

Received: September 23, 2020

Accepted: November 3, 2020

Published: November 6, 2020

Copyright (C) 2020 by author(s) and Scientific Research Publishing Inc. This work is licensed under the Creative Commons Attribution International License (CC BY 4.0).

http://creativecommons.org/licenses/by/4.0/

(c) (i) Open Access

\begin{abstract}
Background: Fahr syndrome associates a set of neuropsychiatric manifestations with bilateral calcifications in the basal ganglia and phosphocalcic disorders. Neuromeningeal cryptococcosis can be present in its manifestations, neuropsychic disorders with or without meningeal signs. The objective was to describe a rare association between Fahr syndrome and neuromeningeal cryptococcosis which can be expressed by the same clinical symptomatology in the context of co-infection with the human immunodeficiency virus (HIV) and the hepatitis B virus (HBV). Presentation: A 37-year-old patient without pathological history, who presented behavioral disorders that led to a fight with those around her and a psychiatric consultation. Then, she was hospitalized in the infectious diseases Department upon discovery of her HIV status and viral hepatitis B. She was logorrheic with behavioral disturbances and subsequently presented with tonic-clonic convulsions. Laboratory tests and imaging have concomitantly discovered Fahr syndrome due to pseudohypoparathyroidism and neuromeningeal cryptococcosis. The correct management of these two pathologies enabled stabilization of the patient's clinical condition with regular monitoring for HIV-HBV coinfection. Conclusion: Farh syndrome and neuromeningeal cryptococcosis are two different entities but sometimes similar symptoms and risk factors. Treatment of metabolic disorders combined with anticryptococcal therapy improved the prognosis.
\end{abstract}




\section{Keywords}

Neuropsychic Disorders, Neuromeningeal Cryptococcosis, Fahr Syndrome, Pseudohypoparathyroidism, HIV

\section{Introduction}

Fahr's syndrome is a set of neuropsychiatric symptoms secondary to the presence of bilateral and symmetrical intracerebral calcifications, non-arteriosclerotic, located in the basal ganglia as well as in the white and gray matter of the brain and cerebellum [1] [2]. Phosphocalcic disorders may explain the clinical picture.

Very few studies have evaluated the prevalence of Farh syndrome. It is quite rare with less than one case in 1,000,000 [3]. On the other hand, neuromeningeal cryptococcosis, an opportunistic pathology in HIV, has a high prevalence in the general population and an incidence of up to $10 \%$ of HIV patients [4] [5]. Clinically, it can also manifest itself in neurological and behavioral disorders [5].

We report a medical observation of a case of Fahr syndrome discovered concomitantly with neuromeningeal cryptococcosis in behavioral disturbances in a patient immunocompromised by HIV.

\section{Case Presentation}

A. C., a 37-year-old female patient, with no neuropsychiatric history, hospitalized for inconsistent speech, psychomotor agitation and aggression in a context of HIV-HBV co-infection. Two weeks earlier, she would have presented behavioral disorders which caused a fight with those around her and then a consultation in the psychiatric department. Complaining subsequently of sharp frontal headaches, nausea, anorexia, she was taken to the emergency room. The finding of weight loss associated with prurigo motivated the performance of a positive retroviral serology (SRV) and allowed its transfer to the infectious diseases department for further treatment. Upon admission, she exhibited logorrhea, visual and auditory hallucinatory, physical and verbal aggression, and thoughts of persecution. The general condition was altered with a body mass index of 14.36 $\mathrm{kg} / \mathrm{m}^{2}$; apyrexia at $37.3^{\circ} \mathrm{C}$; arterial hypotension at $90 / 60 \mathrm{mmHg}$ and tachycardia at 112 beats/min. The neurological examination was without signs of deficit or meningitis with normal consciousness. Brain Computed tomography (CT) scan showed bilateral calcifications of the lenticular nuclei associated with congestive left sphenoidal sinusitis (Figure 1). The blood test performed showed hypocalcemia at $1.93 \mathrm{mmol} / \mathrm{l}(\mathrm{Normal}=2.2-2.6)$, hyperphosphatemia at $1.9 \mathrm{mmol} / \mathrm{l}$ $($ Normal $=0.8-1.6)$ and parathyroid hormone $(\mathrm{PTH})$ normal at $31.02 \mathrm{pg} / \mathrm{ml}$ (Normal $=15-65)$. The other laboratory tests (blood count, serum creatinine, transaminases, C Reactive Protein, protidemia, thyroid hormones) were unremarkable. We started a correction of the hypocalcaemia and 48 hours later, the patient presented generalized tonic-clonic convulsive seizures followed by memory 


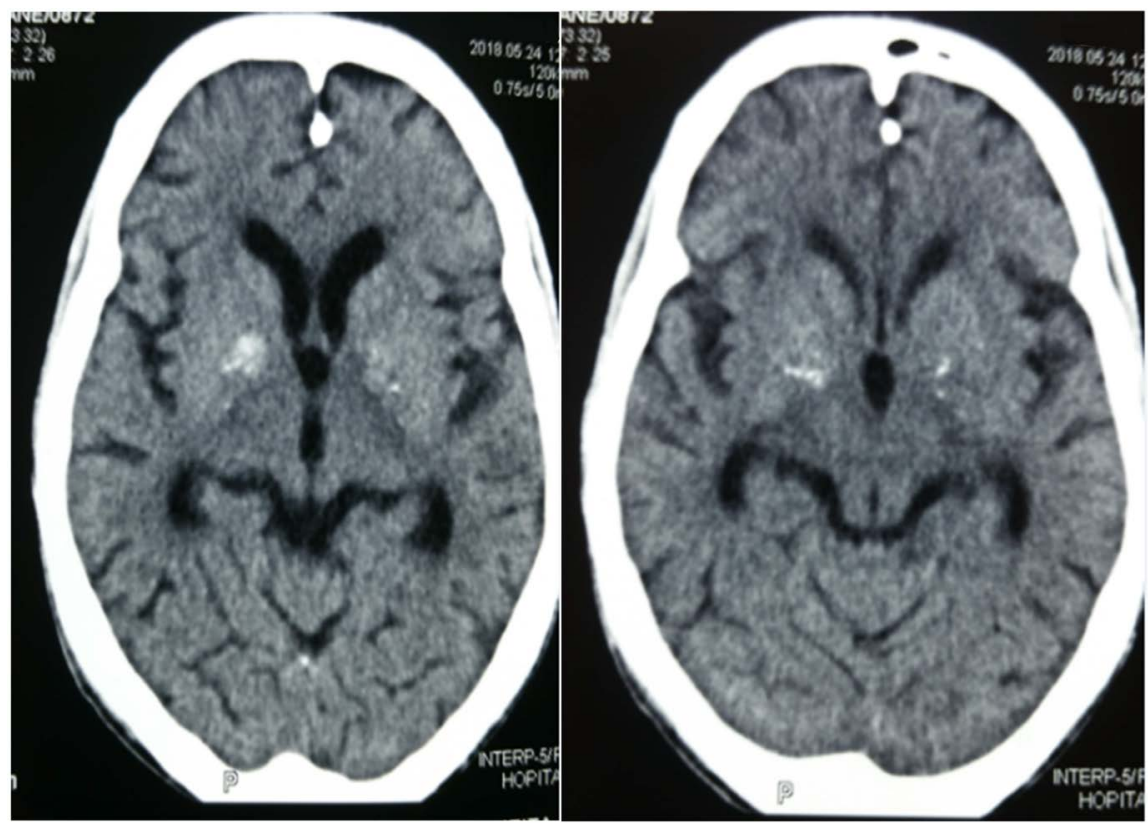

Figure 1. Brain CT-scan showing bilateral calcifications of lenticular nuclei.

disturbances, which motivated an exploratory lumbar puncture of the cerebrospinal fluid (CSF) which revealed a clear fluid and a strong positive agglutination with Pastorex crypto. Retroviral serology was confirmed positive for HIV type 1 with an initial TCD4 lymphocyte count of 17 cells/ $\mu$ l and a viral load of 789,500 copies/ml. The hepatitis B viral markers (HBV) assay revealed progressive chronic hepatitis B with a viral load of 298,263 copies/ml. We concluded that a Fahr syndrome with pseudohypoparathyroidism was associated with neuromeningeal cryptococcosis on HIV-HBV co-infection.

The patient received HAART based on:

- Calci-vitaminotherapy for the correction of metabolic disorders;

- Fluconazole capsule for the treatment of neuromeningeal cryptococcosis;

- Haloperidol for the treatment of neuropsychic disorders;

- Sulfamethoxazole + trimethoprim for chemoprophylaxis against opportunistic infections. The outcome was gradually favorable and four weeks later antiretroviral therapy was started with Tenofovir/lamivudine/efavirenz. The patient returned home after two months of hospitalization. Upon discharge, she was in good clinical condition with amendment of neuropsychic disorders and seizures.

\section{Discussion}

Fahr syndrome is a rare and unrecognized condition [1] [4]. It is most often due to disorders of phosphocalcic metabolism and more particularly in hypoparathyroidism [1] [5] [6] [7]. However, pseudohypoparathyroidism, which is a familial disorder defined by peripheral resistance to the parathyroid hormone, may rarely be the cause, as is the case in our observation [5] [6] [7] [8]. The physiopathological mechanisms which contribute to the occurrence of intracerebral 
calcifications during Fahr syndrome are not well understood. Most authors suggest a metabolic disorder of oligoglial cells with mucopolysaccharide deposits and secondary onset of vascular, perivascular and calcareous lesions [9]. Cases of Fahr syndrome in HIV patients have already been described without any obvious proven relationship [5]. However, bilateral abnormalities of the basal ganglia, especially the lenticular and caudate, during cryptococcosis and of the pallidum in the event of hepatic insufficiency have been reported [10] [11]. Our patient was both infected with HIV, HBV, cryptococcus and probably toxoplasm, suggesting that these infections which are chronic could contribute to intracerebral calcification apart from the phosphocalcic disorders found.

Fahr syndrome is an insidious and progressive condition whose symptoms can appear around the age of 40 to 60 years [1]. However, its association with cryptococcal neuromeningeal infection and immunosuppression may explain the appearance of the signs rather than was the case in our observation. In some case series, the mean age has been estimated at 35 years [5]. In addition, its occurrence in even younger people, especially children, has been described [8] [12] [13]. The clinical symptomatology of Fahr syndrome is polymorphic and nonspecific [5]. The same is true for neuromeningeal cryptococcosis [3]. One can thus find neuropsychic disorders and/or convulsive seizures [3] [8] [12] [14]. This is what we have seen. The diagnosis of Fahr syndrome is based on a brain computed tomography (CT) scan, which identifies calcifications located in the basal ganglia, which may extend to the serrated nuclei of the cerebellum [1] [8] [11] [15]. It is due to the neuropsychic signs that we carried out the cerebral CT scan then the phosphocalcic balance associated with the assay of parathyroid hormone (PTH). It was the addition of seizures despite the correction of hypocalcemia that led us to analyze the LCS and find the presence of cryptococcus. Neuromeningeal cryptococcosis is relatively common, especially in severe HIV immunosuppression [3]. Our patient had a very low CD4 count at 17 cells $/ \mu$ l.

Other conditions, apart from what we encountered in our study, can also give rise to intracerebral calcifications. This is the case for chronic inflammatory pathologies (tuberculosis, CMV infection, flavivirosis, neutrocysticercosis, neurosyphilis), tumor pathologies (astrocytomas, primary cerebral lymphoma), and toxicants (carbon monoxide and lead poisoning, hypervitaminosis $\mathrm{D}$, radiotherapy) [6] [11] [15].

Treatment of Fahr syndrome is based on a symptomatic treatment of the neurological manifestations and calcivitaminotherapy [5]. Treatment of neuromeningeal cryptococcosis is with antifungals, particularly as a first-line treatment and during the induction phase with amphotericin B combined with 5-fluorocytosine [3]. In this case, our patient was treated with fluconazole alone, given its availability and accessibility. The outcome was favorable and after deux months of hospitalization she was discharged of Hospital.

\section{Conclusion}

The particularity of our observation was the concomitant discovery of Fahr syn- 
drome and neuromeningeal cryptococcosis in a case with non-specific symptomatology made up of neuropsychic disorders. The first pathology is rare but the second is an opportunistic infection that is relatively common in severe immunosuppression, most often induced by HIV. In addition, the induction of intracerebral calcification can certainly be found in dysparathyroidism but also in certain infections including neuromeningeal cryptococcosis, cerebral toxoplasmosis and HIV infection as was the case in our observation.

\section{Consent}

The patient gave her informed consent before this study was performed.

\section{Acknowledgements}

Our thanks to the entire team of the Infectious Diseases Department.

\section{Conflicts of Interest}

The authors declare no conflicts of interest regarding the publication of this paper.

\section{References}

[1] Jaworski, K., Styczyńska, M., Mandecka, M., Walecki, J. and Kosior, D.A. (2017) Fahr Syndrome-An Important Piece of a Puzzle in the Differential Diagnosis of Many Diseases. Polish Journal of Radiology, 82, 490-493. https://doi.org/10.12659/PJR.902024

[2] Manyam, B.V. (2005) What Is and What Is Not "Fahr's Disease". Parkinsonism \& Related Disorders, 11, 73-80. https://doi.org/10.1016/j.parkreldis.2004.12.001

[3] Saleem, S., Aslam, H.M., Anwar, M., Anwar, S., Saleem, M., Saleem, A., et al. (2013) Fahr's Syndrome: Literature Review of Current Evidence. Orphanet Journal of Rare Diseases, 8, 156. https://doi.org/10.1186/1750-1172-8-156

[4] Hakim, J., Gangaidzo, I., Heyderman, R., Mielke, J., Mushangi, E. and Taziwa, A. (2000) Impact of HIV Infection on Meningitis in Harare, Zimbabwe: A Prospective Study of 406 Predominantly Adult Patients. AIDS, 14, 1401-1407. https://doi.org/10.1097/00002030-200007070-00013

[5] Shoai Tehrani, M., Charlier-Woerther, C. and Lortholary, O. (2014) Cryptococcosis. EMC-Maladies Infectieuses, 11, 8-613-A-10.

[6] Chouaib, N., Rafai, M., Belkouch, A., Bakkali, H. and Belyamani, L. (2015) Fortuitous Discovery of Fahr's Syndrome after Seizures. Revue Neurologique, 171, 894-895. https://doi.org/10.1016/j.neurol.2015.09.005

[7] Rafai, M.A., Oumari, S., Lytim, S., Boulaajaj, F.Z., El Moutawakkil, B. and Slassi, I. (2014) Fahr's Syndrome: Clinical, Radiological and Etiological Aspects. Feuillets de Radiologie, 54, 2-8. https://doi.org/10.1016/j.frad.2013.10.012

[8] Bonazza, S., La Morgia, C., Martinelli, P. and Capellari, S. (2011) Strio-PallidoDentate Calcinosis: A Diagnostic Approach in Adult Patients. Neurological Sciences, 32, 537-545. https://doi.org/10.1007/s10072-011-0514-7

[9] Sbai, H., Smail, L., Hamdani, S., Essatara, Y., Harrandou, M., Khatouf, M., et al. (2008) Fahr Syndrome Discovered Following a Bacterial Meningitis. La Revue de Médecine Interne, 29, 412-414. https://doi.org/10.1016/j.revmed.2007.11.002 
[10] Moudden, M.K., Zinebi, A., Africha, T. and Baaj, M.E. (2017) Fahr's Syndrome Secondary to Pseudohypoparathyoidism. Médecine thérapeutique, 23, 335-336.

[11] Ouattara-Doumbia, M., Kouassi, L., Kouame-Assouan, A.E., Douayoua-Sonan, T.H. and Boa-Yapo, F. (2006) Maladie de Fahr révélée par des troubles de la marche et de la parole. Revue Internationale des Sciences Médicales d’ Abidjan, 8, 32-35.

[12] Dietemann, J.L. (2018) Neuro-imagerie Diagnostique. 3rd Edition, Elsevier, Paris.

[13] Hegde, A.N., Mohan, S., Lath, N. and Lim, C.C.T. (2011) Differential Diagnosis for Bilateral Abnormalities of the Basal Ganglia and Thalamus. RadioGraphics, 31, 5-30. https://doi.org/10.1148/rg.311105041

[14] Kahloul, N., Chaari, W., Boughamoura, L., Charfeddine, L., Khammeri, S. and Amri, F. (2009) Pseudohypoparathyroidism Revealed by Fahr Syndrome. Archives de Pédiatrie, 16, 444-448. https://doi.org/10.1016/j.arcped.2009.02.017

[15] Billard, C., Dulac, O., Bouloche, J., Echenne, B., Lebon, P., Motte, J., et al. (1989) Encephalopathy with Calcifications of the Basal Ganglia in Children. A Reappraisal of Fahr's Syndrome with Respect to 14 New Cases. Neuropediatrics, 20, 12-19. https://doi.org/10.1055/s-2008-1071258 